\title{
Precision Position Control of Servo Systems Using Adaptive Back-Stepping and Recurrent Fuzzy Neural Networks
}

\author{
Jong Shik Kim, Han Me Kim and Seong Ik Han \\ School of Mechanical Engineering, Pusan National University, \\ Republic of Korea
}

\section{Introduction}

To improve product quality in high-tech industrial fields and in precision product processes, high precision position control systems have been developed. However, high precision position control systems have been faced with a friction problem that exists between the contact surfaces of two materials and produces an obstacle to the precise motion, because the friction is very sensitive to nonlinear time-varying effects such as temperature, lubrication condition, material texture, and contamination degree. Thus, the tracking performance of servo systems can be seriously deteriorated because of the nonlinear friction characteristics.

To overcome the friction problem and to obtain high performance of servo control systems, an appropriate friction model (Olsson, 1998) to describe the nonlinear friction characteristics is required. The LuGre model (Canudas de Wit, 1995) is a representative model. Researchers have used this model because it has a simple structure to be implemented in the design of the controller and can represent most of the friction characteristics except the pre-sliding characteristic.

Model-based control methods for precision position control can be divided into two methods. The first one is the friction feed-forward compensation scheme, which needs the identification of the nonlinear friction phenomena (Olsson, 1998)(Canudas de Wit, 1995). However, it takes a long time and much effort to identify the nonlinear friction. In addition, even with successful completion of the friction identification process, it is difficult to achieve desirable tracking performance due to the nonlinear friction characteristics. Therefore, to achieve desirable tracking performance of servo systems, a robust control scheme should be used simultaneously with the friction feed-forward compensator (Lee, 2004).

The second method is the real time estimation scheme for nonlinear friction coefficients, which is called as the adaptive friction control scheme. This method can actively cope with the variation of the nonlinear friction, which has been proved and studied through experiments (Canudas de Wit, 1997)(Lischinsky, 1999)(Ha, 2000)(Tan, 1999). However, to generate the adaptation rules for the friction coefficients based on the LuGre friction model, a detailed mathematical approach is required. In addition, since the mathematical model of 
the nonlinear friction may include system uncertainties such as unmodeled dynamics, which can cause an undesirable position tracking error of servo systems.

To compensate these system uncertainties and to improve tracking performance, artificial intelligent algorithms such as fuzzy logic and neural networks have been applied because of their advantages to cope with system uncertainties (Wai, 2003)(Leu, 1997)(Peng, 2007)(Lin, 2006). In general, fuzzy logic and neural network algorithms are effective in inferring ambiguous information because of their logicality such as adaptation for learning ability, capacity for experiences, and parallel process ability (Lin, 1996). The fuzzy neural network(FNN) combining the advantages of fuzzy logic and neural network algorithms was presented (Leu, 1997)(Peng, 2007). However, in real applications, the FNN has a static problem due to its feed-forward network characteristics. Therefore, to overcome this static problem of the FNN, the recurrent fuzzy neural network(RFNN) with robust characteristics due to its feed-back structure was presented (Peng, 2007)(Lin, 2006)(Lin, 2004).

In this paper, an adaptive back-stepping control scheme with the RFNN technique is proposed so that servo systems with nonlinear friction uncertainties can achieve higher precision position tracking performance. A dual adaptive friction observer is also designed to observer the internal states of the nonlinear friction model. The position tracking performance of the proposed control system is evaluated through experiments.

The organization of this paper is as follows: In section 2, the dynamic equations for the position servo system with the LuGre friction model are described. In section 3 , to estimate the unknown friction coefficients and to overcome system uncertainties in a position servo system, the adaptive back-stepping controller based on the dual friction observer and the recurrent fuzzy neural networks are designed. In section 4, the experimental results of the tracking performance, the observation of the states, and the estimation of the friction coefficients are shown. Finally, the conclusion is given in section 5.

\section{Modeling of a position servo system}

The layout of a position servo system consists of mass, linear motion guide, ball-screw, and servo motor as shown in Fig. 1. The dynamic equation for the position servo system can be briefly represented as

$$
J \ddot{\theta}=u-T_{f}-T_{d}
$$

where $J$ is the moment of inertia of the servo system, $\ddot{\theta}$ is the angular acceleration of the screw, $u$ is the control input torque, $T_{f}$ is the friction torque, and $T_{d}$ is the disturbance torque due to system uncertainties.

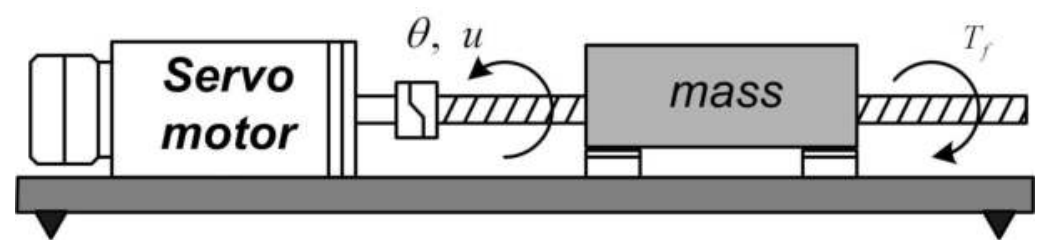

Fig. 1. Layout of the position servo system 
The LuGre model is used for modeling the friction in the position servo system. The LuGre model can describe the nonlinear friction characteristics between two contact surfaces in a mechanical system. As shown in Fig. 2, the relative motion between two contact surfaces can be represented by bristles.

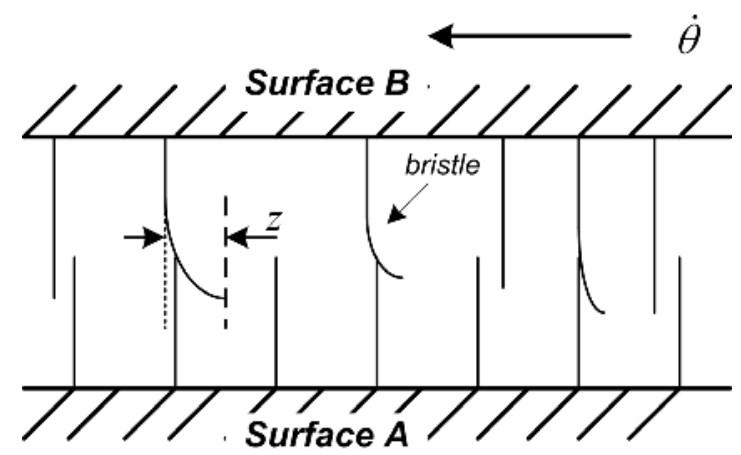

Fig. 2. Friction interfaces with bristles between two surfaces

The stiffness and damping of bristles can be modeled with springs and dampers, respectively. Canudas de Wit represented the average deflection of bristles by a state variable $z$ as follows (Canudas de Wit, 1997) :

$$
\begin{gathered}
\dot{z}=\dot{\theta}-\sigma_{0} h(\dot{\theta}) z, \\
h(\dot{\theta})=\frac{|\dot{\theta}|}{g(\dot{\theta})}
\end{gathered}
$$

where

$$
g(\dot{\theta})=T_{c}+\left(T_{s}-T_{c}\right) e^{-\left(\dot{\theta} / \dot{\theta}_{s t}\right)^{2}}
$$

and $\dot{\theta}$ is the generalized velocity, $\dot{\theta}_{s t}$ is the Stribeck velocity, $\sigma_{0}$ is the nominal static friction parameter, $T_{s}$ is the static friction torque, and $T_{c}$ is the Coulomb friction torque. Also, the friction torque $T_{f}$ was represented as

$$
T_{f}=\mu_{0} z+\mu_{1} \dot{z}+\mu_{2} \dot{\theta}
$$

where $\mu_{0}, \mu_{1}$, and $\mu_{2}$ are the bristle stiffness coefficient, bristle damping coefficient, and viscous damping coefficient, respectively. The function $g(\cdot)$ is assumed to be known and to be a positive value, and it depends on some factors such as material properties and temperature. In order to consider the friction torque variations due to the contact condition of the position servo system, the coefficients $\mu_{0}, \mu_{1}$, and $\mu_{2}$ are assumed to be independent unknown positive constants.

Substituting Eqs. (2), (3), and (4) into Eq. (1), the dynamic equation for the position servo system with friction can be expressed as 


$$
J \ddot{\theta}=u-\mu_{0} z+\mu_{3} h(\dot{\theta}) z-\mu_{4} \dot{\theta}-T_{d}
$$

where

$$
\mu_{3}=\sigma_{0} \mu_{1}, \mu_{4}=\mu_{1}+\mu_{2}
$$

\section{Design of an adaptive control system}

System uncertainties such as high nonlinear friction characteristics according to the operation condition should be considered in precise position servo systems. Thus, feedback linearization and robust control schemes can be considered to reject system nonlinearity and have robustness to unmodeled dynamics, respectively. However, the robust control schemes may not be appropriate for precise position control because these schemes require some premises on bounded uncertainties and bounded disturbance. In addition, if the information on system uncertainties is not included in the control scheme, the feedback linearization scheme may not achieve high precision position tracking performance and make servo systems unstabilize. To overcome these problems in position control servo systems, it is desirable to apply an adaptive control scheme.

\subsection{Design of back-stepping controller}

The back-stepping control(BSC) system can be designed step by step as follows (Krstic, 1995):

Step 1. To achieve the desired tracking performance, the tracking error is defined by the new state $y_{1}$ as

$$
y_{1}=\theta-\theta_{r}
$$

where $\theta_{r}$ is the reference input. The derivative of $y_{1}$ is expressed as

$$
\dot{y}_{1}=\dot{\theta}-\dot{\theta}_{r} .
$$

We define a stabilizing function $\alpha_{1}$ as

$$
\alpha_{1}=\dot{\theta}_{r}-k_{1} y_{1}
$$

where $k_{1}$ is a positive constant. The Lyapunov control function (LCF) $V_{1}$ is selected as

$$
V_{1}=\frac{1}{2} y_{1}^{2}
$$

Then, the derivative of $V_{1}$ is expressed as

$$
\dot{V}_{1}=y_{1} \dot{y}_{1}=y_{1}\left(\dot{\theta}-\alpha_{1}-k_{1} y_{1}\right)=y_{1} y_{2}-k_{1} y_{1}^{2}
$$

where $y_{2}=\dot{\theta}-\alpha_{1}$. 
Step 2. The velocity tracking error is defined by the new state $y_{2}$ as

$$
y_{2}=\dot{\theta}-\alpha_{1}
$$

The derivative of $y_{2}$ can be obtained as

$$
\dot{y}_{2}=\ddot{\theta}-\dot{\alpha}_{1}=\frac{1}{J}\left(u-\mu_{0} z+\mu_{3} h(\dot{\theta}) z-\mu_{4} \dot{\theta}-T_{d}\right)-\dot{\alpha}_{1}
$$

From Eq. (12), in order to select a feedback control law that can guarantee system stability, the LCF for Eq. (11) is selected as

$$
V_{2}=V_{1}+\frac{1}{2} y_{2}^{2}
$$

The derivative of $V_{2}$ can be represented as

$$
\dot{V}_{2}=\dot{V}_{1}+y_{2} \dot{y}_{2}=-k_{1} y_{1}^{2}+y_{2}\left[y_{1}+\frac{1}{J}\left(u-\mu_{0} z+\mu_{3} h(\dot{\theta}) z-\mu_{4} \dot{\theta}-T_{d}\right)-\dot{\alpha}_{1}\right]
$$

If the last term in Eq. (14) is defined as

$$
y_{1}+\frac{1}{J}\left(u-\mu_{0} z+\mu_{3} h(\dot{\theta}) z-\mu_{4} \dot{\theta}-T_{d}\right)-\dot{\alpha}_{1}=-k_{2} y_{2}
$$

where $k_{2}(>0)$ is a design parameter, then the BSC law as the feedback control law can be selected as

$$
u=J\left(-y_{1}-k_{2} y_{2}+\dot{\alpha}_{1}\right)+\mu_{0} z-\mu_{3} h(\dot{\theta}) z+\mu_{4} \dot{\theta}+T_{d}
$$

However, in Eq. (16), the internal state $z$ of the friction model cannot be measured, and friction parameters and the disturbance torque $T_{d}$ cannot be known exactly. In addition, if the friction terms in Eq. (16) cannot be exactly considered in position control servo systems, a large steady-state error may occur.

\subsection{Design of adaptive back-stepping controller and dual friction observer}

In order to select a desired control law, a dual-observer (Tan, 1999) to estimate the unmeasurable internal state $z$ in the friction model is applied as follows:

$$
\begin{aligned}
& \dot{\hat{z}}_{0}=\dot{\theta}-\sigma_{0} h(\dot{\theta}) \hat{z}_{0}+\eta_{0}, \\
& \dot{\hat{z}}_{1}=\dot{\theta}-\sigma_{0} h(\dot{\theta}) \hat{z}_{1}+\eta_{1},
\end{aligned}
$$


where $\hat{z}_{0}$ and $\hat{z}_{1}$ are the estimated values of the internal states in the friction model, and $\eta_{0}$ and $\eta_{1}$ are the observer dynamic terms which can be obtained from an adaptive rule. The corresponding observation errors are given by

$$
\begin{aligned}
& \dot{\tilde{z}}_{0}=-\sigma_{0} h(\dot{\theta}) \tilde{z}_{0}-\eta_{0}, \\
& \dot{\tilde{z}}_{1}=-\sigma_{0} h(\dot{\theta}) \tilde{z}_{1}-\eta_{1},
\end{aligned}
$$

where $\tilde{z}_{0}=z-\hat{z}_{0}$ and $\tilde{z}_{1}=z-\hat{z}_{1}$. Equations (19) and (20) will be induced from the adaptive rule.

In order to induce the adaptive rule to guarantee stability against unknown parameters and the observer dynamic terms, the reconstruction error $E$ is defined as

$$
E=T_{d}-\hat{T}_{d}
$$

where $\hat{T}_{d}$ is the estimated value of $T_{d}$ and it is assumed that $|E| \leq \bar{E}$, where $\bar{E}$ denotes the bounded value of $E$.

We now select the 3rd LCF as follows:

$$
V_{3}=V_{2}+\frac{1}{2 \rho}(\hat{E}-E)^{2}
$$

where $\rho(>0)$ is a positive constant and $\hat{E}$ is the estimated value of the reconstruction error. The derivative of $V_{3}$ can be represented as

$$
\dot{V}_{3}=\dot{V}_{2}+\frac{1}{\rho}(\hat{E}-E) \dot{\hat{E}}=-k_{1} y_{1}^{2}+y_{2}\left[y_{1}+\frac{1}{J}\left(u-\mu_{0} z+\mu_{3} h(\dot{\theta}) z-\mu_{4} \dot{\theta}-T_{d}\right)-\dot{\alpha}_{1}\right]+\frac{1}{\rho}(\hat{E}-E) \dot{\hat{E}}
$$

From Eq. (23), the adaptive back-stepping control(ABSC) law can be selected as

$$
u=J\left(-y_{1}-k_{2} y_{2}+\dot{\alpha}_{1}\right)+\hat{\mu}_{0} \hat{z}_{0}-\hat{\mu}_{3} h(\dot{\theta}) \hat{z}_{1}+\hat{\mu}_{4} \dot{\theta}+\hat{T}_{d}+\hat{E}
$$

Substituting Eq. (24) into Eq. (23), then

$$
\left.\dot{V}_{3}=-k_{1} y_{1}^{2}-k_{2} y_{2}^{2}+\frac{y_{2}}{J}\left[-\mu_{0} \tilde{z}_{0}-\tilde{\mu}_{0} \hat{z}_{0}+\mu_{3} h(\dot{\theta}) \tilde{z}_{1}+\tilde{\mu}_{3} h(\dot{\theta}) \hat{z}_{1}-\tilde{\mu}_{4} \dot{\theta}\right)+\hat{T}_{d}-T_{d}+\hat{E}\right]+\frac{1}{\rho}(\hat{E}-E) \dot{\hat{E}}
$$

where $\tilde{\mu}_{0}=\mu_{0}-\hat{\mu}_{0}, \tilde{\mu}_{3}=\mu_{3}-\hat{\mu}_{3}$, and $\tilde{\mu}_{4}=\mu_{4}-\hat{\mu}_{4}$ are the unknown parameter estimate errors. The 4 th LCF $V_{4}$ is selected as 


$$
V_{4}=V_{3}+\frac{1}{2} \mu_{0} \tilde{z}_{0}^{2}+\frac{1}{2} \mu_{3} \tilde{z}_{1}^{2}+\frac{1}{2 \gamma_{0}} \tilde{\mu}_{0}^{2}+\frac{1}{2 \gamma_{3}} \tilde{\mu}_{3}^{2}+\frac{1}{2 \gamma_{4}} \tilde{\mu}_{4}^{2} .
$$

The derivative of $V_{4}$ can be obtained as

$$
\begin{aligned}
\dot{V}_{4}= & -k_{1} y_{1}^{2}-k_{2} y_{2}^{2}-\mu_{0} \sigma_{0} h(\dot{\theta}) \tilde{z}_{0}^{2}-\mu_{3} \sigma_{0} h(\dot{\theta}) \tilde{z}_{1}^{2}+\tilde{\mu}_{0}\left(-\frac{y_{2}}{J} \hat{z}_{0}-\frac{1}{\gamma_{0}} \dot{\hat{\mu}}_{0}\right)+\tilde{\mu}_{3}\left(\frac{y_{2} h(\dot{\theta})}{J} \hat{z}_{1}-\frac{1}{\gamma_{3}} \dot{\hat{\mu}}_{3}\right) \\
& +\tilde{\mu}_{4}\left(-\frac{y_{2}}{J} \dot{\theta}-\frac{1}{\gamma_{4}} \dot{\hat{\mu}}_{4}\right)+\tilde{z}_{0}\left(-\mu_{0} \frac{y_{2}}{J}-\mu_{0} \eta_{0}\right)+\tilde{z}_{1}\left(\mu_{3} \frac{y_{2}}{J} h(\dot{\theta})-\mu_{3} \eta_{1}\right)+\tilde{E}\left(\frac{y_{2}}{J}+\frac{1}{\rho} \dot{\hat{E}}\right) .
\end{aligned}
$$

From Eq. (27), the update laws can be determined as

$$
\begin{gathered}
\dot{\hat{\mu}}_{0}=-\frac{\gamma_{0}}{J} y_{2} \hat{z}_{0}, \\
\dot{\hat{\mu}}_{3}=\frac{\gamma_{3}}{J} y_{2} h(\dot{\theta}) \hat{z}_{1}, \\
\dot{\hat{\mu}}_{4}=-\frac{\gamma_{4}}{J} y_{2} \dot{\theta},
\end{gathered}
$$

and the observer dynamic terms are expressed as

$$
\begin{gathered}
\eta_{0}=-\frac{y_{2}}{J}, \\
\eta_{1}=\frac{y_{2}}{J} h(\dot{\theta}), \\
\dot{\hat{E}}=-\rho \frac{y_{2}}{J} .
\end{gathered}
$$

Then, Eq. (27) can be represented as

$$
\dot{V}_{4}=-k_{1} y_{1}^{2}-k_{2} y_{2}^{2}-\mu_{0} \sigma_{0} h(\dot{\theta}) \tilde{z}_{0}^{2}-\mu_{3} \sigma_{0} h(\dot{\theta}) \tilde{z}_{1}^{2} \leq-k_{1} y_{1}^{2}-k_{2} y_{2}^{2} \leq 0 .
$$

From Eq. (34), we can define $W(y)$ as follows:

$$
W(y)=k_{1} y_{1}+k_{2} y_{2} \leq-\dot{V}\left(y_{1}, y_{2}\right)
$$

Since $\dot{V} \leq 0, V$ is a non-increasing function. Thus, it has a limit $V_{\infty}$ as $t \rightarrow \infty$. Integrating Eq. (35), then 


$$
\lim _{t \rightarrow \infty} \int_{t_{0}}^{t} W(y(\tau)) d \tau \leq-\lim _{t \rightarrow \infty} \int_{t_{0}}^{t} \dot{V}\left(y_{1}, y_{2}\right) d \tau=\lim _{t \rightarrow \infty}\left\{V\left(y\left(t_{0}\right), t_{0}\right)-V(y(t), t)\right\}=V\left(y\left(t_{0}\right), t_{0}\right)-V_{\infty}
$$

which means that $\int_{t_{0}}^{t} W(y(\tau)) d \tau$ exists and is finite. Since $W(y)$ is also uniformly continuous, the following result can be obtained from Barbalat lemma (Krstic, 1995)(Slotine, 1991) as

$$
\lim _{t \rightarrow \infty} W(y)=0
$$

Since $y_{1}$ and $y_{2}$ are converged to zero as $t \rightarrow \infty, \theta$ and $\dot{\theta}$ approach to $\theta_{r}$ and $\dot{\theta}_{r}$, respectively, as $t \rightarrow \infty$. Therefore, the ABSC system can be asymptotically stable in spite of the variation of system parameters and external disturbance.

\subsection{Design of recurrent fuzzy neural networks}

To determine the lumped uncertainty $T_{d}$, a RFNN observer of a 4-layer structure is proposed, which is shown in Fig. 3. Layer 1 is the input layer with the recurrent loop, which accepts the two input variables. Layer 2 represents the fuzzy rules for calculating the Gaussian membership values. Layer 3 is the rule layer, which represents the preconditions and consequence for the links before and after layer 3, respectively. Layer 4 is the output layer. The interaction and learning algorithms for the layers are given as follows:

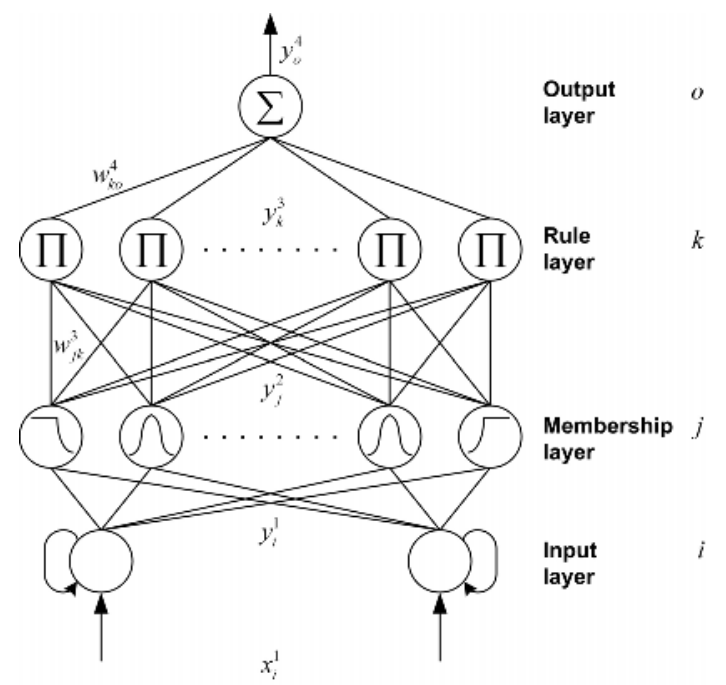

Fig. 3. A general four-layer RFNN

\subsubsection{Description of the RFNN}

Layer 1, Input layer: For each node i, the net input and output are represented, respectively, as 


$$
\begin{gathered}
\operatorname{net}_{i}^{1}=x_{i}^{1}+w_{i}^{1} \cdot y_{i}^{1}(N-1), \\
y_{i}^{1}(N)=f_{i}^{1}\left(\operatorname{net}_{i}^{1}(N)\right)=\operatorname{net}_{i}^{1}(N), i=1,2
\end{gathered}
$$

where $x_{1}^{1}=y_{1}, x_{2}^{1}=\dot{y}, w_{i}^{1}$ is the recurrent weights, and $N$ denotes the number of iterations.

Layer 2, Membership layer: For each node, the Gaussian membership values are calculated. For the $j$ th node,

$$
\begin{gathered}
\operatorname{net}_{j}^{2}(N)=-\frac{\left(x_{i}^{2}-m_{i j}\right)^{2}}{\left(\sigma_{i j}\right)^{2}} \\
y_{j}^{2}(N)=f_{j}^{2}\left(\operatorname{net}_{j}^{2}(N)\right)=\exp \left(\operatorname{net}_{j}^{2}(N)\right), j=1, \ldots, n
\end{gathered}
$$

where $m_{i j}$ and $\sigma_{i j}$ are the mean and standard deviation of the Gaussian function in the jth term of the ith input linguistic variable $x_{i}^{2}$ to the node of layer 2, respectively. $n$ is the total number of the linguistic variables with respect to the input nodes.

Layer 3, Rule layer: Each node $\mathrm{k}$ in this layer is denoted by $\Pi$. In addition, the input signals in this layer are multiplied each other and then the result of the product is generated.

$$
\begin{gathered}
\operatorname{net}_{k}^{3}(N)=\prod_{j} w_{j k}^{3} x_{j}^{3}(N), \\
y_{k}^{3}(N)=f_{k}^{3}\left(\operatorname{net}_{k}^{3}(N)\right)=\operatorname{net}_{k}^{3}(N), k=1, \ldots, l
\end{gathered}
$$

where $x_{j}^{3}$ represents the jth input to the node of layer $3, w_{j k}^{3}$ is the weights between the membership layer and the rule layer. $l=(n / i)^{i}$ is the number of rules with complete rule connection, if each input node has the same linguistic variables.

Layer 4, Output layer: The single node o in this layer is labeled as $\Sigma$, which computes the overall output as the summation of all input signals:

$$
\begin{gathered}
\operatorname{net}_{o}^{4}(N)=\sum_{k} w_{k o}^{4} x_{k}^{4}(N), \\
y_{o}^{4}(N)=f_{o}^{4}\left(\operatorname{net}_{o}^{4}(N)\right)=\operatorname{net}_{o}^{4}(N)
\end{gathered}
$$

where the connecting weight $w_{k o}^{4}$ is the output action strength of the oth output associated with the kth rule. $x_{k}^{4}$ represents the kth input to the node of layer 4 , and $y_{o}^{4}=\hat{T}_{d}$.

\subsubsection{On-line learning algorithm}

In the learning algorithm, it is important to select parameters for the membership functions and weights to decide network performance. In order to train the RFNN effectively, on-line 
parameter learning is executed by the gradient decent method. There are four adjustable parameters. Our goal is to minimize the error function $e$ represented as

$$
e=\frac{1}{2}\left(\theta_{r}-\theta\right)^{2}=\frac{1}{2}\left(y_{1}\right)^{2}
$$

By using the gradient descent method, the weight in each layer is updated as follows:

Layer 4: The weight is updated by an amount

$$
\Delta w_{k o}^{4}=-\eta_{w} \frac{\partial e}{\partial w_{k o}^{4}}=\left(-\eta_{w} \frac{\partial e}{\partial u} \frac{\partial u}{\partial \operatorname{net}_{o}^{4}}\right)\left(\frac{\partial \text { net }_{o}^{4}}{\partial w_{k o}^{4}}\right)=\eta_{w} y_{1} x_{k}^{4}
$$

where $y_{1}=-\frac{\partial e}{\partial u} \frac{\partial u}{\partial \text { net }_{o}^{4}}$ and $\eta_{w}$ is the learning-rate parameter of the connecting weights of the RFNN.

Layer 3: Since the weights in this layer are unified, the approximated error term needs to be calculated and propagated to calculate the error term of layer 2 as follows:

$$
\delta_{k}^{3}=-\frac{\partial e}{\partial \operatorname{net}_{k}^{3}}=-\frac{\partial e}{\partial u} \frac{\partial u}{\partial \operatorname{net}_{o}^{4}} \frac{\partial \text { net }_{o}^{4}}{\partial y_{k}^{3}} \frac{\partial y_{k}^{3}}{\partial \operatorname{net}_{k}^{3}}=y_{1} w_{k o}^{4}
$$

Layer 2: The multiplication operation is executed in this layer by using Eq. (46). To update the mean of the Gaussian function, the error term is computed as follows:

$$
\delta_{j}^{2}=-\frac{\partial e}{\partial n e t_{j}^{2}}=-\frac{\partial e}{\partial u} \frac{\partial u}{\partial \text { net }_{o}^{4}} \frac{\partial \text { net }_{o}^{4}}{\partial y_{k}^{3}} \frac{\partial y_{k}^{3}}{\partial \text { net }_{k}^{3}} \frac{\partial \text { net }_{k}^{3}}{\partial y_{j}^{2}} \frac{\partial y_{j}^{2}}{\partial \text { net }_{j}^{2}}=\sum_{k} \delta_{k}^{3} y_{k}^{3}
$$

and then the update law of $m_{i j}$ is

$$
\Delta m_{i j}=-\eta_{m} \frac{\partial e}{\partial m_{i j}}=-\eta_{m} \frac{\partial e}{\partial y_{j}^{2}} \frac{\partial y_{j}^{2}}{\partial \operatorname{net}_{j}^{2}} \frac{\partial \operatorname{net}_{j}^{2}}{\partial m_{i j}}=\eta_{m} \delta_{j}^{2} \frac{2\left(x_{i}^{2}-m_{i j}\right)}{\sigma_{i j}^{2}}
$$

where $\eta_{m}$ is the learning-rate parameter of the mean of the Gaussian functions. The update law of $\sigma_{i j}$ is

$$
\Delta \sigma_{i j}=-\eta_{s} \frac{\partial e}{\partial \sigma_{i j}}=-\eta_{s} \frac{\partial e}{\partial y_{j}^{2}} \frac{\partial y_{j}^{2}}{\partial \operatorname{net}_{j}^{2}} \frac{\partial \operatorname{net}_{j}^{2}}{\partial \sigma_{i j}}=\eta_{s} \delta_{j}^{2} \frac{2\left(x_{i}^{2}-m_{i j}\right)^{2}}{\sigma_{i j}^{3}}
$$


where $\eta_{s}$ is the learning-rate parameter of the standard deviation of the Gaussian functions.

The weight, mean, and standard deviation of the hidden layer can be updated by using the following equations:

$$
\begin{gathered}
w_{k o}^{4}(N+1)=w_{k o}^{4}+\Delta w_{k o}^{4} \\
m_{i j}(N+1)=m_{i j}(N)+\Delta m_{i j} \\
\sigma_{i j}(N+1)=\sigma_{i j}(N)+\Delta \sigma_{i j}
\end{gathered}
$$

\section{Experiment results}

Figure 4 shows the servo position tracking control system to evaluate the performance of control schemes. The angular position was measured with an incremental rotary encoder whose counts per encoder was 4 times of 10000 pulses per revolution. A data acquisition board with D/A 12-bit resolution was used to supply the driving voltage to the motor. The sampling rate of the servo system was selected as $500 \mathrm{~Hz}$. The control algorithms were programmed with C-language. The parameters of the servo system and friction model for experiment are shown in Table 1. The block diagram of the ABSC system with RFNN is shown in Fig. 5.

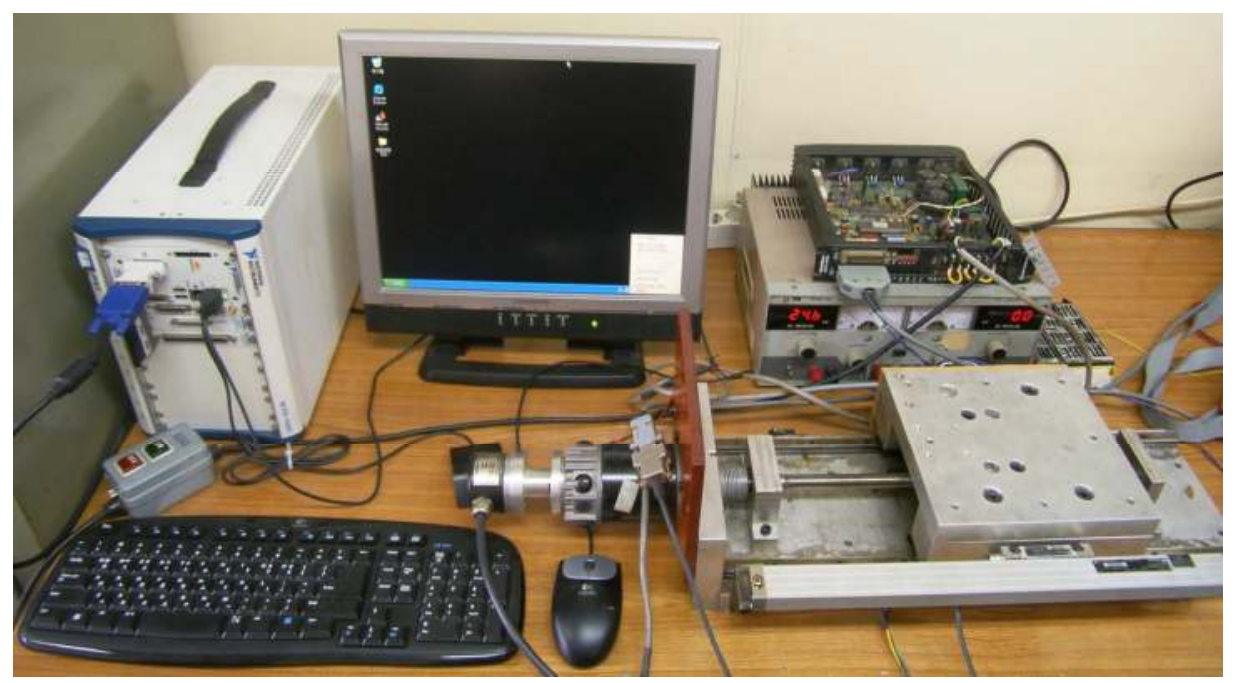

Fig. 4. Photograph of the servo position tracking control system 


\begin{tabular}{|c|c|c|}
\hline Parameter & Notation & Value \\
\hline Moment of inertia & $J$ & $2.3 \times 10^{-5} \mathrm{kgm}^{2}$ \\
\hline Bristles stiffness coefficient & $\sigma_{0}$ & $0.15 \mathrm{Nm}$ \\
\hline Stribeck velocity & $\dot{\theta}_{\text {st }}$ & $0.013 \mathrm{rad} / \mathrm{s}$ \\
\hline Coulomb friction & $T_{c}$ & $1.97 \times 10^{-3} \mathrm{Nm}$ \\
\hline Static friction & $T_{s}$ & $2.6 \times 10^{-3} \mathrm{Nm}$ \\
\hline
\end{tabular}

Table 1. Parameters of the servo and friction model

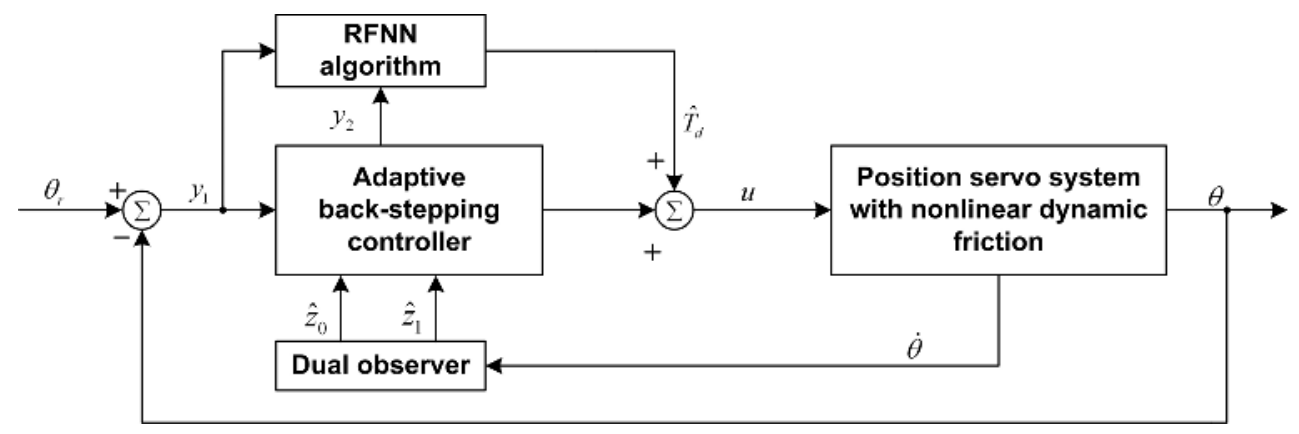

Fig. 5. Block diagram of the ABSC system with RFNN

In order to evaluate the performance of the servo system with the proposed control scheme, two reference inputs were applied as follows:

$$
\begin{gathered}
\theta_{r_{1}}=0.1 \sin (0.4 \pi t)[\mathrm{rad}] \\
\theta_{r_{2}}=0.1 \sin (0.125 \pi t) \sin (0.75 \pi t)[\mathrm{rad}]
\end{gathered}
$$

To compare the tracking performances of the BSC system, ABSC system, ABSC system with RFNN, the reference input $\theta_{r_{1}}$ was continuously used for experiment as follows: the BSC system was applied during the initial 20 seconds, the ABSC system during the 40 seconds after the application of the BSC system, and the ABSC system with RFNN during the 40 seconds after the application of the ABSC system. The reference input $\theta_{r_{2}}$ was independently experimented for the ABSC system and the ABSC system with RFNN, respectively. In addition, the structure of the RFNN is defined to two neurons at inputs of which each has the recurrent loop, five neurons at the membership layer, five neurons at the rule layer, and one neuron at the output layer. The fuzzy sets at the membership layer, which have the mean $\left(m_{i j}\right)$ and standard deviation $\left(\sigma_{i j}\right)$, were determined according to the maximum variation boundaries of $y_{1}$ and $y_{2}$ of the ABSC system without RFNN. $m_{i j}$ and $\sigma_{i j}$ vectors applied to experiment are selected as follows:

$$
m_{1 j}=[-0.002,-0.001,0.0,0.001,0.002] \times \kappa_{1},
$$




$$
\begin{gathered}
m_{2 j}=[-0.2,-0.1,0.0,0.1,0.2] \times \kappa_{2}, \\
\sigma_{1 j}=[0.003,0.003,0.003,0.003,0.003] \times \kappa_{3}, \\
\sigma_{2 j}=[0.3,0.3,0.3,0.3,0.3] \times \kappa_{4}
\end{gathered}
$$

where $m_{1 j}$ and $\sigma_{1 j}$ indicate the mean and standard deviation vectors of $y_{1}$, respectively, $m_{2 j}$ and $\sigma_{2 j}$ indicate the mean and standard deviation vectors of $y_{2}$, respectively, and $\kappa_{i}=1,(i=1,2,3,4)$.

Figure 6 shows the error of the BSC system, ABSC system, and ABSC system with RFNN for the reference input $\theta_{r_{1}}$. The angular displacement rms(root mean square) error of the BSC system is 0.0054. While the ABSC system is operating, its maximum error tends to exponentially decrease and then converge to a steady state value due to $\hat{\sigma}_{0}, \hat{\sigma}_{3}$, and $\hat{\sigma}_{4}$ by the update rules which are given by Eqs. (52), (53), and (54). The angular displacement rms error of the ABSC system is 0.0027. In the operating range of the ABSC system with RFNN, the angular displacement error converges to a steady state value after experiencing a transient state for about 1 second because of the switch from the ABSC system to the ABSC system with RFNN. The angular displacement rms error is 0.0005 . The tracking performance of the ABSC system compared with it of the BSC system is improved by 2 times and it of the ABSC system with RFNN compared with it of the ABSC system is improved by 5.4 times. The performance improvement of the ABSC system with RFNN implies that the control input of the RFNN including the reconstruction estimation compensates system uncertainties.

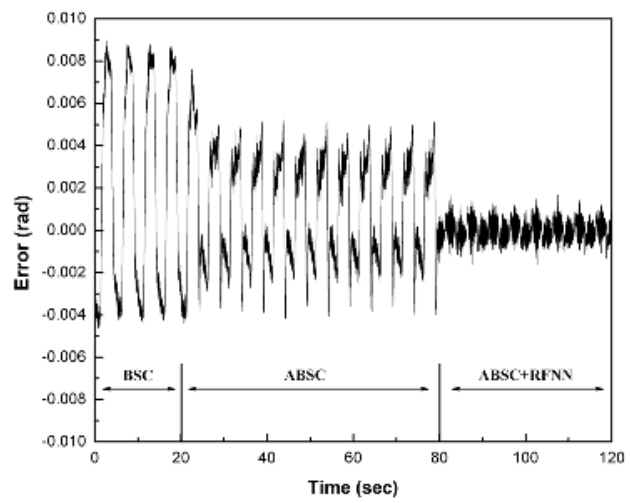

Fig. 6. Error of the BSC system, ABSC system, and ABSC system with RFNN for the reference input $\theta_{r_{1}}$

Figure 7 shows the estimation and the observation of the BSC system, ABSC system, and ABSC system with RFNN for the reference input $\theta_{r_{1}}$. The estimations by the update rule are shown in Fig. 7(a). The BSC system estimates the friction parameter to be 0, because the BSC system does not have the update rule for $\hat{\sigma}_{0}, \hat{\sigma}_{3}$, and $\hat{\sigma}_{4}$. When the ABSC system is applied to the servo system, the update rules estimates the friction parameters, which converge to 
some values; this convergence stabilizes the servo position system. When the ABSC system is switched to the ABSC system with RFNN, the estimations of the friction parameters do not vary because the angular displacement error is largely decreased by the RFNN. Therefore, the friction estimation values can maintain steady state in the operating range where the RFNN is used. Figure 7(b) shows the observations of the dual observer. The spike phenomenon of $\hat{z}_{0}$ among both observation values is occurred to a changing point of velocity, because $y_{2}$ corresponds to the velocity error, which directly affects $\hat{z}_{0}$, as described in Eq. (31). However, in the case of the ABSC system with RFNN, the spike phenomenon of $\hat{z}_{0}$ is largely removed, which means that the RFNN compensates system uncertainties such as nonlinear friction including Coulomb friction, static friction, Stribeck velocity, and unmodeled dynamics.

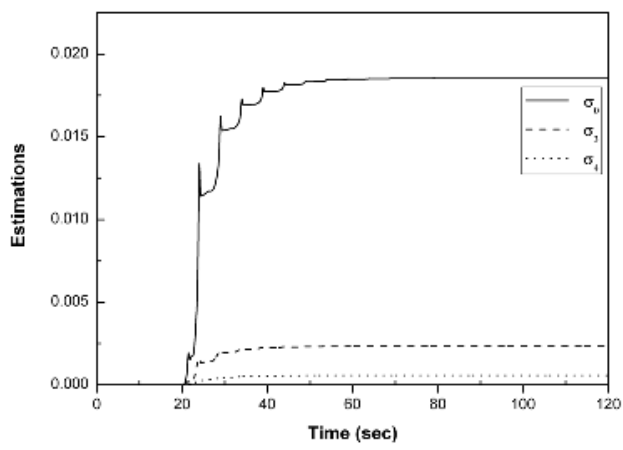

(a) Estimations of the update rule

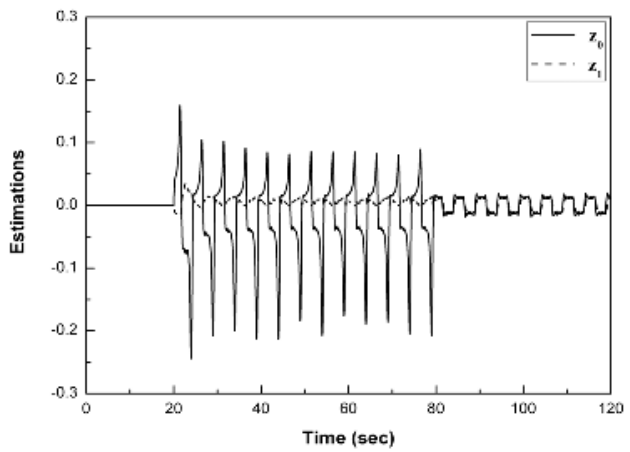

(b) $z_{0}$ and $z_{1}$ of the dual observer

Fig. 7. Estimation and observation of the BSC system, ABSC system, and ABSC system with RFNN for the reference input $\theta_{r_{1}}$

Figure 8 shows the estimated friction torque of the BSC system, ABSC system, and ABSC system with RFNN for the reference input $\theta_{r_{1}}$. The estimated friction torques of the BSC system, ABSC system, and ABSC system with RFNN reflect the results of Fig. 7. Figure 9 shows the control input of the BSC system, ABSC system, and ABSC system with RFNN for the reference input $\theta_{r_{1}}$. When the RFNN including reconstruction error estimation is 


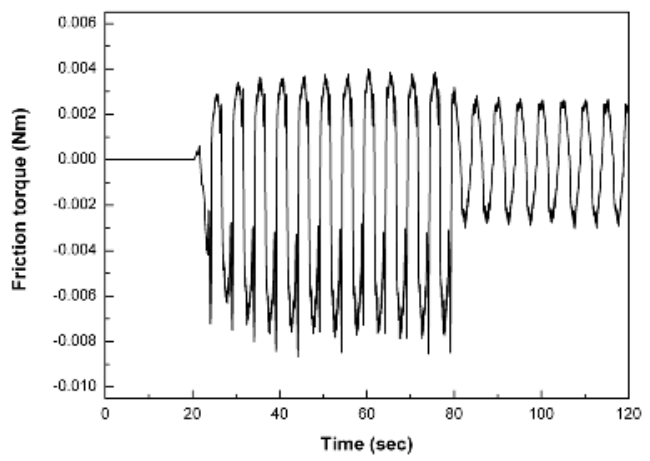

Fig. 8. Estimated friction torque of the BSC system, ABSC system, and ABSC system with RFNN for the reference input $\theta_{r_{1}}$

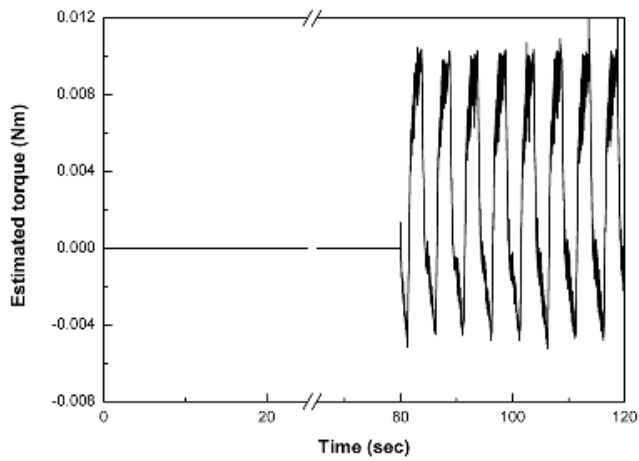

(a) Estimated torque of the RFNN including the reconstruction error

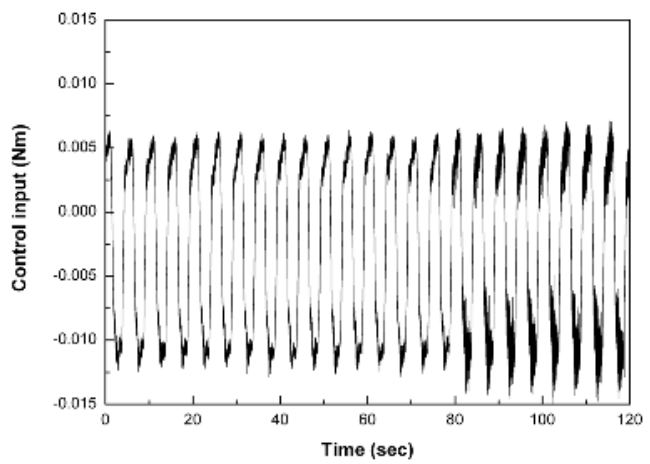

(b) Control input torque applied to the servo system

Fig. 9. Control inputs of the BSC system, ABSC system, and ABSC system with RFNN for the reference input $\theta_{r_{1}}$

applied to the servo system at 80 seconds as shown in Fig. 9(a), a little more control input than before that is required to compensate system uncertainties as shown in Fig. 9(b). In 
addition, the deflection of the control input removes the deflection of the error for the BSC and ABSC systems, which is shown in Fig. 6.

Figure 10 shows the errors of the ABSC system and ABSC system with RFNN for the reference input $\theta_{r_{2}}$. The reference input $\theta_{r_{2}}$ reflects a real situation and includes more system uncertainties because of the time varying amplitude sinusoidal input. In addition, the experiment conditions of the ABSC system and ABSC system with RFNN are all the same. The tracking error rms values of the ABSC system with RFNN and ABSC system are 0.0007 and 0.003 , respectively. Therefore, the tracking rms error of the ABSC system with RFNN is four times less than that of the ABSC system, which implies that the RFNN is suitable for compensating system uncertainties.

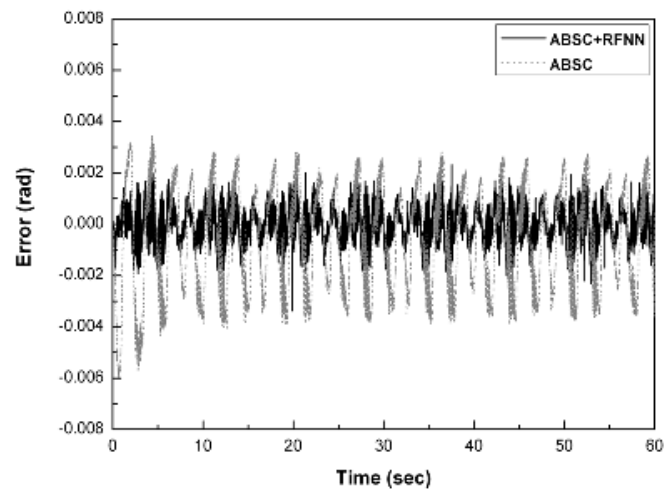

Fig. 10. Errors of the ABSC system, and ABSC system with RFNN for the reference input $\theta_{r_{2}}$

Figure 11 shows the friction parameter estimations for the ABSC system and ABSC system with RFNN for the reference input $\theta_{r_{2}}$. The estimations of the friction parameters converge to steady state values in about 20 seconds as shown in Fig. 11(a). The estimation values of the friction parameters for the ABSC system with RFNN are much smaller than those for the ABSC system, as shown in Fig. 11(b), because the RFNN and the reconstruction error estimator rapidly decrease the tracking error by reducing system uncertainties.

Figure 12 shows the estimated friction torques of the ABSC system and ABSC system with RFNN for the reference input $\theta_{r_{2}}$. The parameters of the ABSC system with RFNN were estimated to be approximately 0 , because the RFNN compensated system uncertainties including nonlinear friction. Therefore, the effectiveness of the RFNN was clearly demonstrated from the above results.

Figure 13 shows the control input of the ABSC system and ABSC system with RFNN for the reference input $\theta_{r_{2}}$. The estimated torque of the RFNN including the reconstruction error and the control input torque applied to the servo motor are shown in Figs. 13(a) and (b), respectively. The ABSC system with RFNN generated a little more control input than the ABSC system due to the estimation result of the RFNN including the reconstruction error, as shown in Fig. 13(a). This implies that the ABSC system with RFNN compensates system uncertainties such as nonlinear friction and unmodeled dynamics, satisfactorily. 


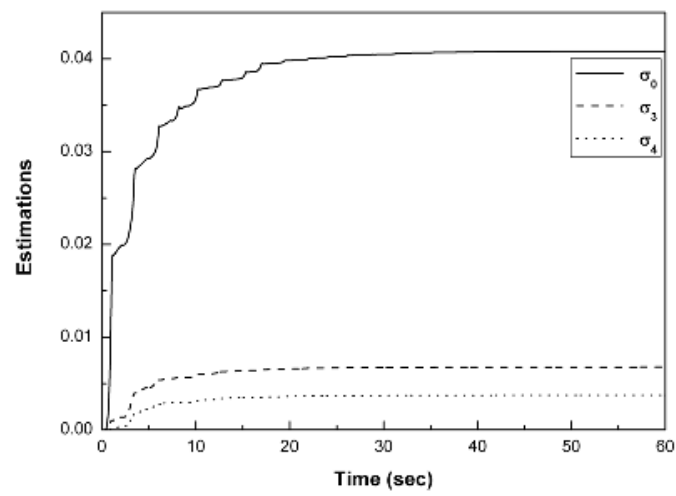

(a) Estimation of the adaptive rule of the ABSC system

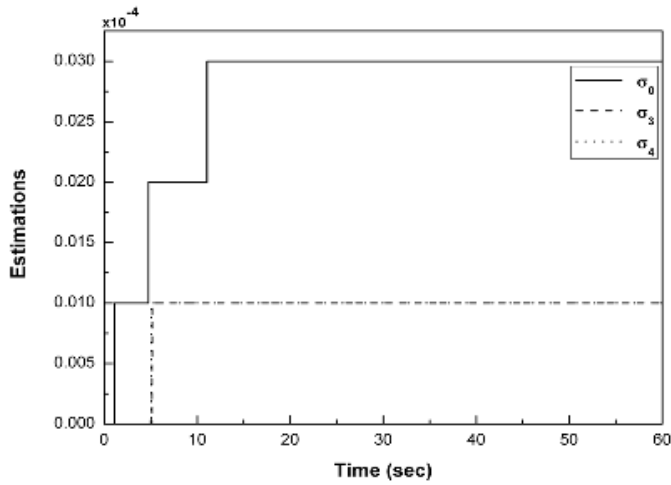

(b) Estimation of the adaptive rule of the ABSC system with RFNN

Fig. 11. Friction parameter estimations of the ABSC system and ABSC system with RFNN for the reference input $\theta_{r_{2}}$

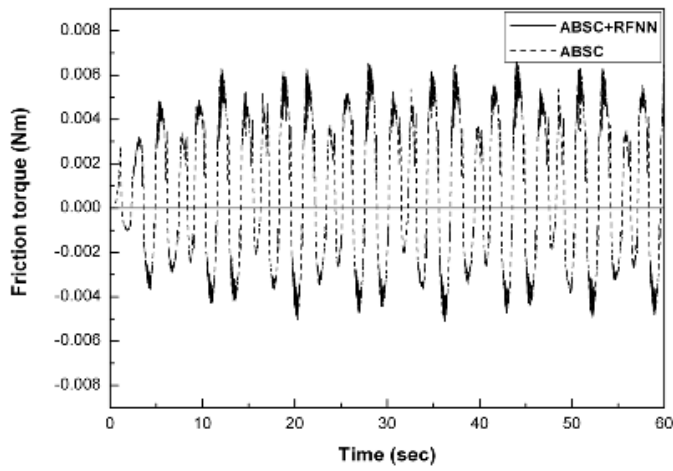

Fig. 12. Estimated friction torques of the ABSC system and ABSC system with RFNN for the reference input $\theta_{r_{2}}$ 


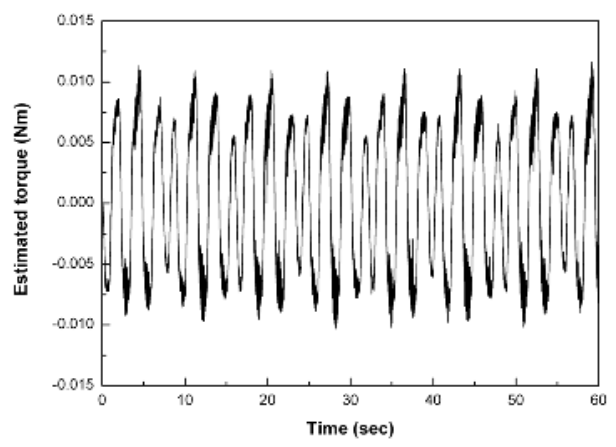

(a) Estimated torque of the RFNN including the reconstruction error

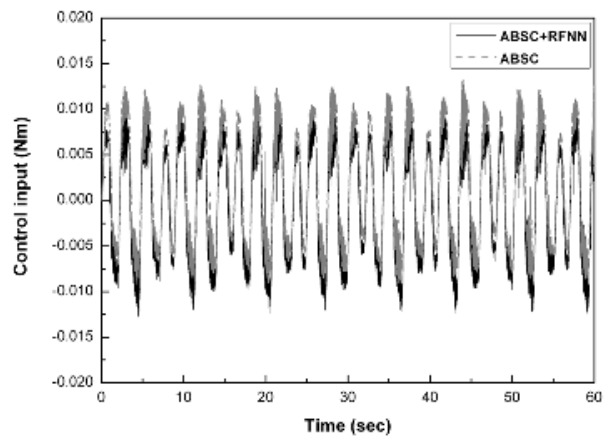

(b) Control input torque applied to the servo system

Fig. 13. Control input of the ABSC system and ABSC system with RFNN for the reference input $\theta_{r_{2}}$

In order to show an influence of the RFNN parameters on control performance, two main parameters, which are $m_{i j}$ and $\sigma_{i j}$ of the Gaussian fuzzy membership function in Layer 2, are changed. Initial values of these values are selected by investigating the range and magnitude of $y_{1}$ and $y_{2}$, and then there are on-line updated through Eqs. (53) and (54). On the other hand, the change in the weight factors is not considered to experimental condition because of using initial random values.

Figure 14 shows the results of the ABSC system with the variation of $m_{i j}$ and $\sigma_{i j}$ in RFNN for the reference input $\theta_{r_{2}}$. The changed conditions of the mean and standard deviation are $\kappa_{i}=0.5$ and $\kappa_{i}=1.5$. For $\kappa_{i}=0.5$, the results of the error, estimation, and estimated friction torque of the ABSC system with RFNN are diverged due to the reduction of $m_{i j}$ and $\sigma_{i j}$ in 7.5 seconds as shown in Fig. 14 (a), (b), and (c). On the other hand, although the error state of the ABSC system with RFNN for $\kappa_{i}=1.5$ is stable as shown in Fig. 14(a), the angular displacement rms error of compared system with the ABSC system with RFNN in Fig. 10 is minutely increased to 1.25 times. In addition, although the estimations of the adaptive rule of the ABSC system with RFNN as shown in Fig. 14(b) compared with their estimation values as shown in Fig. 11(b) is increased, their effect for the estimated friction torque is very 
small as shown in Fig. 14(c) compared with their estimated friction torque of the ABSC system as shown in Fig. 12, which reflects the result of Fig. 14(b). At this time, the ratio of the maximum friction torque in Fig. 12 to it in Fig. 14(c) is approximately 30 times. Thus, we can conclude that $m_{i j}$ and $\sigma_{i j}$ of the Gaussian membership function in the RFNN depend on the error output of the servo system. Finally, $m_{i j}$ and $\sigma_{i j}$ of the Gaussian membership function in the RFNN need to be carefully selected.

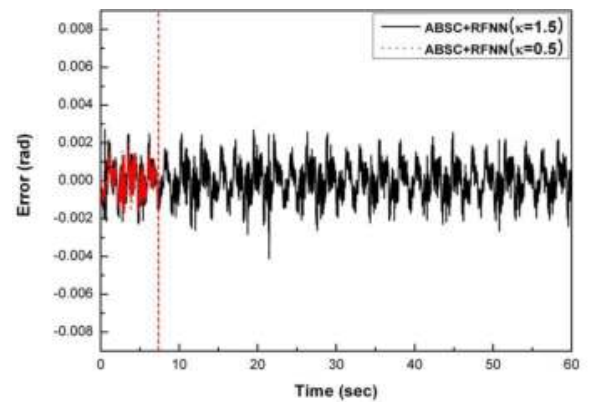

(a) Error of the ABSC system with RFNN

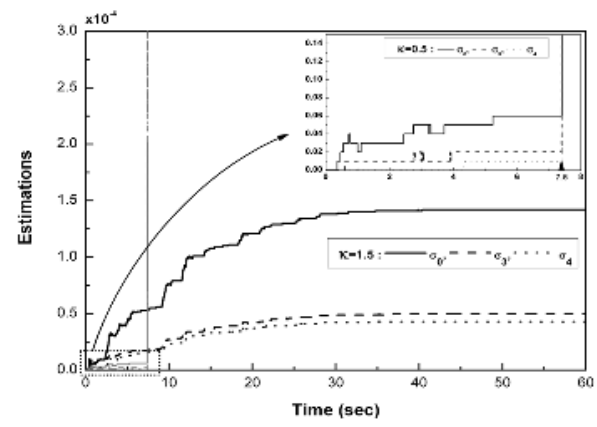

(b) Estimation of the adaptive rule of the ABSC system with RFNN

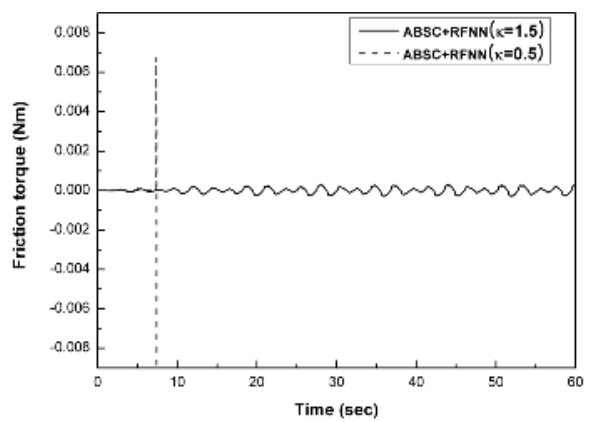

(c) Estimated friction torque of the ABSC system with RFNN

Fig. 14. Results of the ABSC system with the variation of $m_{i j}$ and $\sigma_{i j}$ in RFNN for the reference input $\theta_{r_{2}}$ 


\section{Conclunsion}

The tracking performance of servo systems is deteriorated by nonlinear friction and system uncertainties, especially in the region where the direction of velocity of servo systems is changed. In order to reduce the effects of the friction and system uncertainties, a robust adaptive precision position control scheme is proposed. Unmeasurable state and parameters of the dynamic friction model are observed and estimated by the dual observer and the adaptive back-stepping controller, respectively. In order to actively cope with system uncertainties, the RFNN scheme is applied to the servo system. Experiments showed that the servo system with the dual observer, adaptive back-stepping controller, and RFNN including the reconstruction error estimator can achieve desired tracking performance and robustness. In addition, the influence of the mean and standard deviation of the RFNN parameters on control performance is shown through experiment.

\section{References}

C. Canudas de Wit and P. Lischinsky (1997), Adaptive Friction Compensation with Partially Known Dynamic Friction Model, Int. J. Adaptive Control and Signal Processing, 11, 65-80.

C. Canudas de Wit, H. Olsson, and P. Lischinsky (1995), A New Model for Control of Systems with Friction. IEEE Trans. Automatic Control, 40(3), 419-425.

C. H. Lin (2004), Adaptive Recurrent Fuzzy Neural Network Control for Synchronous Reluctance Motor Servo Drive, IEE Proc. Electr. Power Appl., 151(6), 711-724.

C. T. Lin, and C. S. Greorge (1996), Neural Fuzzy Systems, Prentice-Hall PTR, New Jersey, USA.

F. J. Lin, S. L. Yang and P. H. Shen(2006), Self-Constructing Recurrent Fuzzy Neural Network for DSP-Based Permanent-Magnet Linear-Synchronous-Motor Servodrive, IEE Proc. Electr. Power Appl., 153(2), 236-246.

H. Olsson, K. J. Astrom, C. C. Wit, M. Gafvert and P. Lischinsky (1998), Friction Models and Friction Compensation, Eur. J. Control, 4(3), 176-185.

J. J. Slotine and W. Li (1991), Applied Nonlinear Control, Pearson Education, New Jersey, USA.

J. Z. Peng, Y. N. Wang, W. Sun (2007), Trajectory-Tracking Control for Mobile Robot Using Recurrent Fuzzy Cerebellar Model Articulation Controller, Neural Inform ProcessLetters \& Rev, 11(1), 15-23.

K. J. Lee, H. M. Kim, and J. S. Kim (2004), Design of a Chattering-Free Sliding Mode Controller with a Friction Compensator for Motion Control of a Ball-Screw System, Proc. ImechE Part-I, Journal of Systems and Control Engineering, 218 (5), 369-380.

M. Krstic, I. Kanellakopoulos and P. Kokotovic (1995), Nonlinear and Adaptive Control Design, Wiley Interscience, New York, USA.

P. Lischinsky, C. Canudas de Wit, and G. Morel (1999), Friction Compensation for an Industrial Hydraulic Robot, IEEE Contr. Syst. Mag., 19, 25-32.

Q. R. Ha, D. C. Rye, and H. F. Durrant-Whyte(2000), Variable Structure Systems Approach to Friction Estimation and Compensation. Proc. of IEEE, Int. Control on Robot. $\mathcal{E}$ Auto, 3543-3548.

R. J. Wai (2003), Robust Fuzzy Neural Network Control for Nonlinear Motor-Toggle Servomechanism, Fuzzy Sets and Systems, 139, 185-208.

Y. G. Leu, T. T. Lee and W. Y. Wang(1997), On-Line Turning of Fuzzy-Neural Networks for Adaptive Control of Nonlinear Dynamic Systems, IEEE Trans. System Man Cybern, 27(6), 1034-1043.

Y. Tan, and I. Kanellakopoulos (1999), Adaptive Nonlinear Friction Compensation with Parametric Uncertainties. Proc. AACC, 2511-2515. 


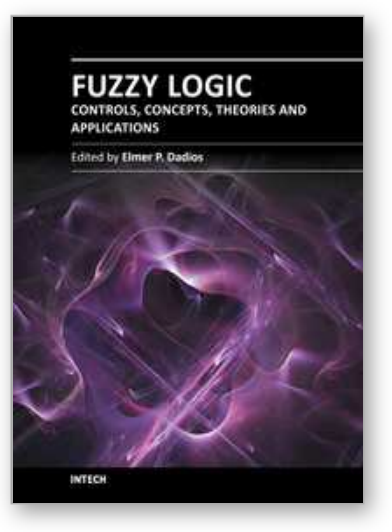

\author{
Fuzzy Logic - Controls, Concepts, Theories and Applications \\ Edited by Prof. Elmer Dadios
}

ISBN 978-953-51-0396-7

Hard cover, 428 pages

Publisher InTech

Published online 28, March, 2012

Published in print edition March, 2012

This book introduces new concepts and theories of Fuzzy Logic Control for the application and development of robotics and intelligent machines. The book consists of nineteen chapters categorized into 1) Robotics and Electrical Machines 2) Intelligent Control Systems with various applications, and 3) New Fuzzy Logic Concepts and Theories. The intended readers of this book are engineers, researchers, and graduate students interested in fuzzy logic control systems.

\title{
How to reference
}

In order to correctly reference this scholarly work, feel free to copy and paste the following:

Jong Shik Kim, Han Me Kim and Seong Ik Han (2012). Precision Position Control of Servo Systems Using Adaptive Back-Stepping and Recurrent Fuzzy Neural Networks, Fuzzy Logic - Controls, Concepts, Theories and Applications, Prof. Elmer Dadios (Ed.), ISBN: 978-953-51-0396-7, InTech, Available from:

http://www.intechopen.com/books/fuzzy-logic-controls-concepts-theories-and-applications/precision-positioncontrol-of-servo-systems-using-adaptive-back-stepping-and-recurrent-fuzzy-neural-

\section{INTECH}

open science | open minds

\section{InTech Europe}

University Campus STeP Ri

Slavka Krautzeka 83/A

51000 Rijeka, Croatia

Phone: +385 (51) 770447

Fax: +385 (51) 686166

www.intechopen.com

\section{InTech China}

Unit 405, Office Block, Hotel Equatorial Shanghai

No.65, Yan An Road (West), Shanghai, 200040, China

中国上海市延安西路 65 号上海国际贵都大饭店办公楼 405 单元

Phone: +86-21-62489820

Fax: +86-21-62489821 
(C) 2012 The Author(s). Licensee IntechOpen. This is an open access article distributed under the terms of the Creative Commons Attribution 3.0 License, which permits unrestricted use, distribution, and reproduction in any medium, provided the original work is properly cited. 